\title{
Sex differences in the risk profile and male predominance in silent brain infarction in community- dwelling elderly subjects: the Sefuri brain MRI study
}

\author{
Yuki Takashima $^{1}$, Yoshikazu Miwa ${ }^{2}$, Takahiro Mori ${ }^{1}$, Manabu Hashimoto ${ }^{1}$, Akira Uchino ${ }^{3}$, Takefumi Yuzuriha ${ }^{1}$, \\ Toshiyuki Sasaguri $^{2}$ and Hiroshi Yao ${ }^{1}$
}

Although brain infarction is more common in men, the male predominance of silent brain infarction (SBI) was inconsistent in the earlier studies. This study was to examine the relationship between sex differences in the risk profile and SBI. We conducted a population-based, cross-sectional analysis of cardiovascular risk factors and SBI on MRI. We asked all the female participants about the age at natural menopause and parity. SBI was detected in $77(11.3 \%)$ of 680 participants $(266$ men and 414 women) with a mean age of 64.5 (range 40-93) years. In the logistic analysis, age (odds ratio (OR) $=2.760 / 10$ years, $95 \%$ confidence interval $(\mathrm{Cl})=2.037-3.738)$, hypertension $(\mathrm{OR}=3.465,95 \% \mathrm{Cl}=1.991-6.031)$, alcohol intake $(\mathrm{OR}=2.494,95 \%$ $\mathrm{Cl}=1.392-4.466)$ and smoking $(\mathrm{OR}=2.302,95 \% \mathrm{Cl}=1.161-4.565)$ were significant factors concerning $\mathrm{SBI}$. Although $\mathrm{SBI}$ was more prevalent among men, this sex difference disappeared on the multivariate model after adjustment for other confounders. In $\mathbf{2 1 5}$ women aged $\mathbf{6 0}$ years or older, age at natural menopause, early menopause, duration of menopause, number of children and age at the last parity were not significantly associated with SBI after adjustment for age. Hypertension and age were considered to be the major risk factors for SBI in community-dwelling people. Male predominance in SBI was largely due to higher prevalence of alcohol habit and smoking in men than in women in our population.

Hypertension Research (2010) 33, 748-752; doi:10.1038/hr.2010.69; published online 30 April 2010

Keywords: asymptomatic stroke; lacunar infarction; menopause; MRI; risk factors

\section{INTRODUCTION}

Brain infarction is more common in men. ${ }^{1}$ Male sex may be a risk factor for symptomatic stroke, whereas premenopausal women appeared to be protected from cardiovascular events or stroke. Exceptions were groups of 35-44 years and over 85 years, in which women had slightly greater stroke incidence than men. ${ }^{2}$ Estrogen may have beneficial effects on endothelial function and atherosclerosis in addition to the hormone's effects on serum lipid concentrations, raising the possibility of sex differences in arterial remodeling. ${ }^{3}$ The ability to have children at older age may be a marker for slow aging and extreme longevity. ${ }^{4}$ On the contrary, Zhang et al. ${ }^{5}$ have found that high gravity and high parity were associated with a higher risk of ischemic stroke. In the Framingham Heart Study, early menopause was associated with an increased incidence of ischemic stroke. ${ }^{6}$ Taken together, female sex may be a non-modifiable protective factor against symptomatic ischemic stroke. However, the male predominance of silent brain infarction (SBI) was inconsistent in the earlier studies. ${ }^{7-11}$

With regard to SBI, the North Manhattan Study found that male sex was independently associated with SBI on a multivariable logistic regression model. ${ }^{7}$ Although the Rotterdam Scan Study reported a higher prevalence of SBI among women than men, the sex difference was no longer statistically significant when adjusted for other risk factors. ${ }^{8}$ In a population-based consecutive autopsy series of residents (the Hisayama Study), SBI tended to be more frequent (non-significant on multivariate analysis) in female among 713 subjects without clinical stroke: $16.2 \%$ of 390 men and $19.2 \%$ of 323 women with a mean age at death of 78.3 years. ${ }^{9}$ In contrast, sex was not independently associated with an increased risk of SBI in the Cardiovascular Health Study and Framingham Offspring Study. ${ }^{10,11}$ In this study, we conducted a population-based, cross-sectional analysis of brain MRI findings to examine the relationship between sex differences in the risk profile and SBI.

\section{METHODS}

Between 1997 and 2007, we randomly contacted approximately 1200 inhabitants aged 40 years or older, living in the rural community of Sefuri village, Saga, Japan, through the village office, and 720 subjects (60\%) visited for their first MRI examination. These subjects were living independently at home

${ }^{1}$ Center for Emotional and Behavioral Disorders, National Hospital Organization Hizen Psychiatric Center, Saga, Japan; 2Department of Clinical Pharmacology, Graduate School of Medical Sciences, Kyushu University, Fukuoka, Japan and ${ }^{3}$ Department of Radiology, Saitama Medical University International Medical Center, Saitama, Japan

Correspondence: Dr H Yao, Center for Emotional and Behavioral Disorders, National Hospital Organization Hizen Psychiatric Center, Mitsu 160, Yoshinogari, Kanzaki, Saga 842-0192, Japan.

E-mail: hyao@hizen2.hosp.go.jp

Received 25 January 2010; revised 3 March 2010; accepted 17 March 2010; published online 30 April 2010 
without apparent dementia. Eight subjects did not undergo MRI examination because of claustrophobia $(n=5)$ and contraindications for MRI $(n=3)$. Subjects with a history of stroke $(n=18)$, brain tumor $(n=5)$, malignant neoplasm $(n=2)$, psychiatric disorders including depression $(n=3)$ or a history of head trauma $(n=4)$ were also excluded. Two cases of transient ischemic attack were included in subjects with a history of stroke: one had progressed to overt stroke 2 days later and the other proved to be a small putamenal hemorrhage on MRI. The National Hospital Organization Hizen Psychiatric Center Institutional Review Board approved the study (No. 15-1), and written informed consent was obtained from all subjects.

Participants underwent a structured clinical interview, a neurological examination, general hematology tests, biochemistry tests and ECGs. Blood pressure was measured in the sitting position by the standard cuff method after a 5-min rest. Vascular risk factors were defined as described earlier. ${ }^{12,13}$ Briefly, arterial hypertension was considered present if a subject had a history of repeated blood pressure recordings above $140 / 90 \mathrm{~mm} \mathrm{Hg}$ or the subject was being treated for hypertension. Diabetes mellitus was defined as fasting plasma glucose $>7.77 \mathrm{mmoll}^{-1}$ and/or $\mathrm{HbAlc}>6.0 \%$, or an earlier diagnosis of diabetes mellitus. Hyperlipidemia was defined as total serum cholesterol concentration $>5.69 \mathrm{mmoll}^{-1}$ or if the subject was being treated for hyperlipidemia. We obtained information about usual alcohol intake and type of alcohol consumed from a detailed questionnaire as described earlier. ${ }^{13}$ We defined one drink as $10 \mathrm{~g}$ of ethanol, calculated as follows: $350 \mathrm{ml}$ beers as 1.4 drinks, $180 \mathrm{ml}$ sake (rice wine) as 2.2 drinks, $180 \mathrm{ml}$ shochu (white spirits) as 3.6 drinks, $60 \mathrm{ml}$ whisky as 2.0 drinks and $120 \mathrm{ml}$ wine as 1.2 drinks. In this study, we defined alcohol intake as one drink or more per week, because the earlier study from the same population revealed that even light drinkers had the similar risk for SBI (odds ratio $(\mathrm{OR})=4.1,95 \%$ confidence interval $(\mathrm{CI})=1.7-10.0)$ as moderate drinkers $(\mathrm{OR}=3.1,95 \% \mathrm{CI}=1.3-7.0)$. Former drinkers were considered non-drinker in this study. Smoking was defined as present if the subject smoked at least an average of 10 cigarettes per day.

We asked all the female participants about items discussed below using a questionnaire, and analyzed potential risk factors for SBI in 215 women aged 60 years or older in relation to age at natural menopause and parity. Natural menopause was considered to occur if a woman had ceased menstruating naturally for at least 1 year. Age at natural menopause was the self-reported age at last menstrual period. Early menopause was defined as natural menopause before age 40 . Women were also queried as to the cause of menopause (natural, surgical, other), whether a hysterectomy was performed, number of ovaries removed, the use of hormone replacement therapy, the total number of children and the age at giving birth to her last child. Subjects with non-natural menopause were excluded from the analysis.

The combination of T1WI, T2WI and FLAIR images is required to accurately detect both SBI and white matter lesions (WMLs). ${ }^{14}$ Therefore, T1 weighted $(\mathrm{TR} / \mathrm{TE}=510 / 12 \mathrm{~ms})$, T2 weighted $(\mathrm{TR} / \mathrm{TE}=4300 / 110 \mathrm{~ms})$ and FLAIR $(\mathrm{TR} / \mathrm{TI} / \mathrm{TE}=6750 / 1600 / 22 \mathrm{~ms})$ images were obtained with a slice thickness of $6 \mathrm{~mm}$ with a $1 \mathrm{~mm}$ interslice gap with an MRI (1.0 T, Shimadzu Magnex XP, Kyoto, Japan). SBI was shown as low signal intensities on T1-weighted images, and their size was $5 \mathrm{~mm}$ or larger as described earlier (Figure 1). ${ }^{12,13}$ We differentiated enlarged perivascular spaces from SBI on the basis of their location, shape and size. ${ }^{15,16}$ The WMLs were defined as isointense with normal brain parenchyma on T1-weighted images, high signal intensity areas on T2-weighted images and were classified into deep white matter lesions (DWMLs) and periventricular hyperintensities (PVHs). We used the validated rating scale of DWMLs by Fazekas et al. ${ }^{17}$ : Grade 0, absent; Grade 1, punctate foci; Grade 2, beginning confluence of foci; Grade 3, large confluent areas.

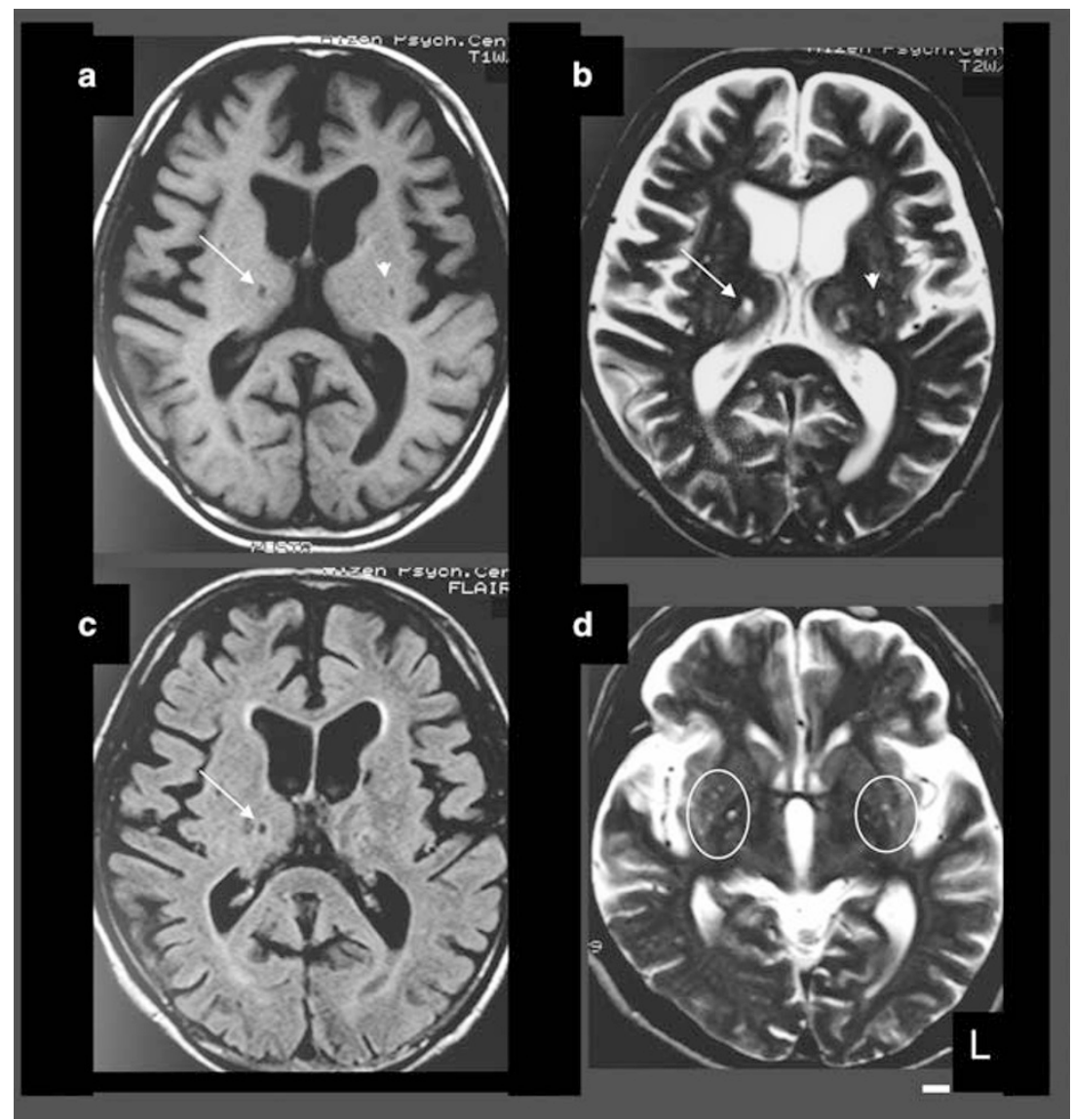

Figure 1 (a-c) An 80-year-old man with silent brain infarction (SBI) in the right thalamus (arrow) and the left basal ganglia (arrow head). The SBI is shown as low signal intensity on the T1 weighted (A), high signal intensity on the T2 weighted and iso or high signal intensity with or without low intensity at the center on the fluid-attenuated inversion recovery (FLAIR) images. (d) A 70-year-old man with dilated periventricular spaces in the bilateral lower one-third of basal ganglia (circles). Bar indicates $10 \mathrm{~mm}$. L, left. 
For PVHs, we determined the presence and severity (Grade 0 , absent; Grade 1, pencil thin; Grade 2, smooth halo lining) using FLAIR images. All scans were reviewed independently by two authors (HY and AU) who were blinded to all clinical data. In the case of disagreement between the raters, a consensus reading was held.

All values were given as mean \pm s.d. The data were analyzed with the Predictive Analysis Software (PASW Statistics 18.0, formerly called SPSS Statistics, SPSS Inc., Chicago, IL, USA). A significance level of 0.05 was used in all analyses. For the univariate analysis, the $t$-test for continuous variables, the $\chi^{2}$ test for categorical variables and the non-parametric Mann-Whitney $U$-test for variables with skewed distribution were used as appropriate. We chose the variables for entry into the multivariate analysis based on the clinical and neuroradiological findings after univariate testing. Multivariate analysis was performed using the forward stepwise method of logistic analysis.

\section{RESULTS}

The subjects comprised 266 men and 414 women with a mean age of 64.5 (range 40-93) years (Table 1). SBI, DWMLs and PVHs were detected in $77(11.3 \%), 204(30.0 \%)$ and $121(17.8 \%)$ of 680 participants, respectively. The prevalence of all these MRI findings increased steeply with age, whereas SBI alone were more frequent in men than in women (Figure 2). Of 77 subjects with SBI, 73 (95\%) had only lacune(s) and 51 of 73 subjects $(70 \%)$ had a single lacune.

The mean age at natural menopause was $48.7 \pm 4.4$ years (a median of 50 years), and the mean number of children was $3.5 \pm 1.4$ (a median of 3). Age at natural menopause, early menopause (10 of 215 women) and age at the last parity were not significantly associated with SBI (Table 2). Although duration of menopause $(P=0.010)$ and number of children $(P=0.052)$ tended to be associated with SBI on univariate analysis, these associations did not persist after adjustment for age. In this group of female subjects aged 60 years or older, alcohol habit was independently associated with SBI. Even if hypertension, diabetes mellitus and hyperlipidemia were forced into the multivariate model regardless of statistical significance, they did not affect the results of Table 2.

In the logistic analysis, age $(\mathrm{OR}=2.760 / 10$ years, $95 \% \mathrm{CI}=2.037-$ 3.738), hypertension ( $\mathrm{OR}=3.465,95 \% \mathrm{CI}=1.991-6.031)$, alcohol intake $(\mathrm{OR}=2.494,95 \% \mathrm{CI}=1.392-4.466)$ and smoking $(\mathrm{OR}=2.302$, $95 \% \mathrm{CI}=1.161-4.565)$ were significant factors concerning SBI (Table 3). Male sex was not significantly associated with WMLs. Hypertension and age, but neither alcohol nor smoking, were the major factors associated with both DWMLs and PVHs (Table 3).

Table 1 Sex differences in demographic measures

\begin{tabular}{lccc}
\hline & All & Men & Women \\
& $\mathrm{n}=680$ & $\mathrm{n}=266$ & $\mathrm{n}=414$ \\
\hline Age (years) & $64.5 \pm 11.5$ & $64.3 \pm 11.0$ & $64.6 \pm 11.8$ \\
Education (years) & $10.1 \pm 2.4$ & $10.5 \pm 2.6$ & $9.7 \pm 2.3$ \\
Body mass index (kg m ${ }^{-2}$ ) & $23.0 \pm 3.2$ & $23.0 \pm 3.2$ & $22.9 \pm 3.3$ \\
Hypertension (\%) & 38.2 & 37.6 & 38.6 \\
Systolic blood pressure (mm Hg) & $138 \pm 23$ & $135 \pm 21$ & $141 \pm 24^{*}$ \\
Diastolic blood pressure (mm Hg) & $79 \pm 11$ & $81 \pm 10$ & $78 \pm 11^{* *}$ \\
Mean blood pressure (mm Hg) & $99 \pm 13$ & $99 \pm 12$ & $99 \pm 13$ \\
Diabetes mellitus (\%) & 9.6 & 15.4 & $5.8^{* *}$ \\
Hyperlipidemia (\%) & 23.8 & 19.9 & $31.9^{* *}$ \\
Alcohol (\%) & 39.1 & 74.1 & $16.7^{* *}$ \\
Smoking (\%) & 16.3 & 38.7 & $1.9 * *$ \\
\hline Values are mean \pm s.d. & & & \\
* $P=0.002,{ }^{* *} P<0.001$ vs. $\mathrm{men}$. & & &
\end{tabular}

Although SBI was more prevalent among men on univariate analysis $(P=0.015)$, this sex difference disappeared on the multivariate model after adjustment for other confounders. Age, body mass index, hypertension and blood pressure were well balanced between men and women (Table 1). Hypertension was present in $260(38.2 \%)$ of 680 subjects. Blood pressure levels were $126 \pm 16 / 76 \pm 9,153 \pm 20$ / $81 \pm 11$ and $166 \pm 17 / 89 \pm 10 \mathrm{~mm} \mathrm{Hg}$ in normotensive subjects $(n=420)$, treated hypertensive subjects $(n=169)$ and non-treated hypertensive subjects $(n=91)$, respectively. Common vascular risk factors such as diabetes mellitus, alcohol and smoking were more frequent in men, and more frequent hyperlipidemia in women.

\section{DISCUSSION}

This is the first study, which showed that higher prevalence of lifestyle risk factors rather than sex explains the male predominance of SBI. We found a prevalence of SBI of $11.3 \%$ in community-dwelling subjects with a mean age of 64.5 years. This is comparable with two prior studies, the Atherosclerosis Risk in Community (ARIC) Study ${ }^{18}$ and the Framingham Heart Study ${ }^{11}$ (prevalence and mean age: 11\%, 63 years and $10.7 \%, 61$ years, respectively). A Japanese brain check-up study, among participants who received MRI at their own expense, reported a similar prevalence of $10.6 \%{ }^{19}$ This study showed that age, hypertension, alcohol habit and smoking were independently associated with SBI. We found a male predominance of SBI (15.0\% in men vs. $8.9 \%$ in women), which was similar to the North Manhattan Study (21.3\% in men vs. $15.2 \%$ in women). ${ }^{7}$ However, male sex was not significantly associated with SBI after adjustment for other vascular risk factors. In other words, higher prevalence of risk factors such as alcohol and smoking in men produced male predominance of SBI in this study.
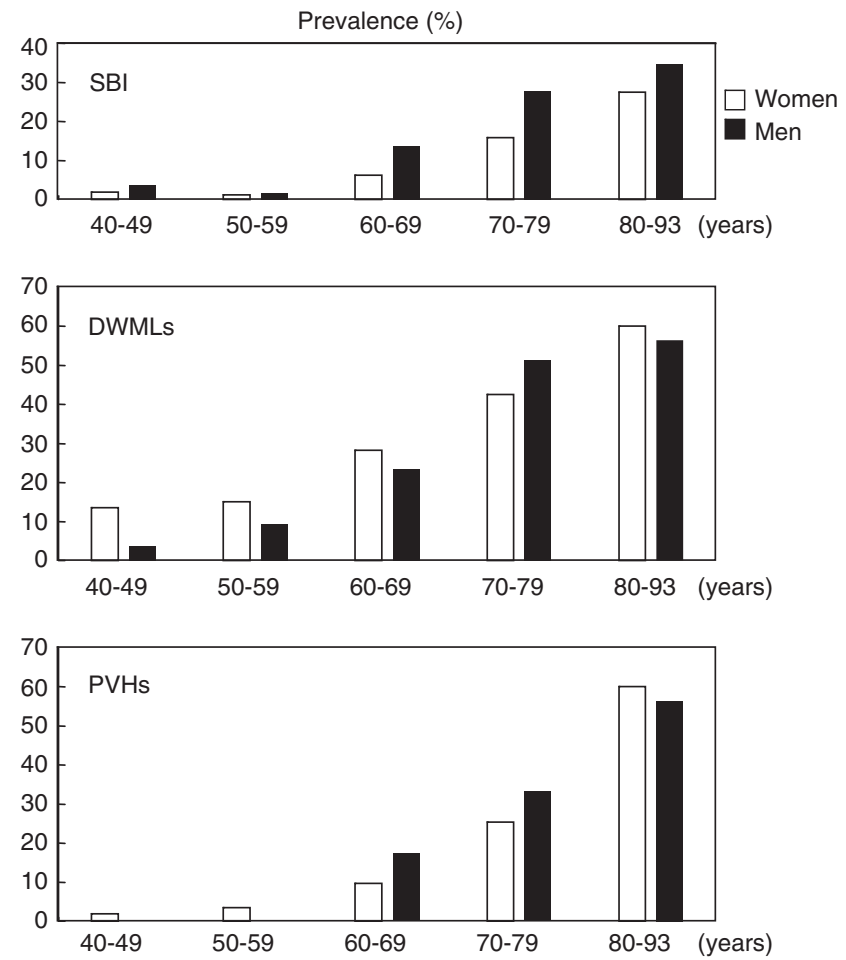

Figure 2 Prevalence of silent brain infarction (SBI), deep white matter lesions (DWMLs) and periventricular hyperintensities (PVHs) with increasing age in male and female participants. 
In this study, assumed risk factors unique to women such as early menopause did not associate with SBI, and older age at the last parity, as a potential marker for longevity, seemed negative in terms of preventing SBI in women. Age at natural menopause, early menopause and age at the last parity were not significantly associated with SBI. Duration of menopause and number of children, which tended to be associated with SBI on univariate analysis, were not significant after adjustment for age. As the majority of silent infarcts are related to small vessel disease, menopause and parity might not be detected as factors relevant to SBI. Alternatively, the age at menopause and parity might be an important risk for atherosclerosis (for example largeartery occlusive infarction, carotid atherosclerosis and coronary heart disease). ${ }^{20-22}$ Strengths of this study include that it is population based and includes a relatively large number of residents. The limitation of this study would be that we could not provide the sex differences in incidence of SBI because of the cross-sectional nature. The Rotterdam Scan Study, which reported higher prevalence of SBI among women than men, ${ }^{8}$ showed similar SBI incidence for both sexes. ${ }^{23}$ Sex differences in SBI incidence need to be further investigated in future studies. Another limitation of this study would be that women before age 60 were excluded from the analysis for the effects of natural menopause and parity on SBI, because SBI was rare before 60 years (2 of 139 women). Therefore, we cannot exclude the

Table 2 Menopause, parity and silent brain infarction among women aged 60 years or older

\begin{tabular}{lcccc}
\hline & & \multicolumn{3}{c}{ Multivariate } \\
\cline { 3 - 5 } & Univariate & Odds ratio & $95 \% \mathrm{Cl}$ & $\mathrm{P}$ \\
\hline Age (per 10 years) & 0.004 & 2.573 & $1.395-4.746$ & 0.002 \\
Alcohol & 0.081 & 3.475 & $1.070-11.285$ & 0.038 \\
Age at natural menopause & 0.506 & & & \\
Early menopause & 0.119 & & & \\
Duration of menopause & 0.010 & & & \\
(per 10 years) & & & & \\
No. of children & 0.052 & & & \\
Age at the last parity & 0.149 & & & \\
\hline
\end{tabular}

Abbreviation: $\mathrm{Cl}$, confidence interval. possibility that early menopause or high parity could be the basis for SBI at younger ages.

Age and hypertension are the most widely accepted risk factors for SBI, and other cardiovascular risk factors for symptomatic stroke were also found to raise the risk of SBI. ${ }^{24}$ In the longitudinal Rotterdam Study, age, blood pressure, diabetes mellitus, cholesterol, homocysteine levels and smoking were associated with new SBI in participants without prevalent infarcts. ${ }^{23}$ Similarly, the Framingham Offspring Study showed that atrial fibrillation, hypertension, systolic blood pressure and an elevated plasma homocysteine, but neither age nor gender were independently associated with an increased risk of SBI. ${ }^{11}$ As most of silent infarcts are subcortical lacunar infarction, risk factors for SBI are similar to those of lacunar infarction or the small vessel disease. Recently, the community-based PATH Through Life Study showed that hypertension was the major treatable risk factor for lacunar infarcts. ${ }^{25}$ Therefore, the present results are compatible with those reported earlier in terms of risk factor profiles of SBI.

Two earlier studies, the Cardiovascular Health Study and the ARIC Study, have investigated the effects of alcohol intake on subclinical MRI findings. ${ }^{26,27}$ The Cardiovascular Health Study found that moderate alcohol consumption in older adults, aged 65 years or older was associated with a lower prevalence of SBI, whereas the ARIC Study showed that alcohol intake in middle-aged adults was not associated with MRI infarction. Our earlier study as well as this study revealed that even a low amount of regular drinking may be a risk factor for SBI in community-dwelling Japanese people. ${ }^{13}$ Effects of alcohol on SBI were evident also in the selected group of female subjects with natural menopause aged 60 years or older (Table 3 ). Discrepancies between the results of our study and those of the earlier studies may be partly explained by racial differences such as obviously lower body mass index of Japanese compared with those of western populations, and the frequent genetic deficiency in alcohol detoxification in Japanese and Orientals. ${ }^{28}$ Furthermore, among a Japanese population, lacunar infarction was the most common subtype of cerebral infarction, ${ }^{29}$ whereas stroke registries of western countries have reported lower frequencies of lacunar infarction than of atherothrombotic and cardioembolic infarction. Alcohol may be partly protective against proximal segment of the cerebrovascular tree, but not for small vessels or SBI.

The relationship between smoking and SBI has been unclear. With regard to symptomatic stroke, an early meta-analysis revealed that the

Table 3 Potential risk factors for silent brain infarction and white matter lesions

\begin{tabular}{|c|c|c|c|c|c|c|c|c|c|c|c|c|}
\hline & \multicolumn{4}{|c|}{ Silent brain infarction } & \multicolumn{4}{|c|}{ Deep white matter lesions } & \multicolumn{4}{|c|}{ Periventricular hyperintensities } \\
\hline & \multirow{2}{*}{$\begin{array}{c}\text { Univariate } \\
\mathrm{P}\end{array}$} & \multicolumn{3}{|c|}{ Multivariate } & \multirow{2}{*}{$\begin{array}{c}\text { Univariate } \\
\mathrm{P}\end{array}$} & \multicolumn{3}{|c|}{ Multivariate } & \multirow{2}{*}{$\begin{array}{c}\text { Univariate } \\
\mathrm{P}\end{array}$} & \multicolumn{3}{|c|}{ Multivariate } \\
\hline & & Odds ratio & $95 \% \mathrm{Cl}$ & $P$ & & Odds ratio & $95 \% \mathrm{Cl}$ & $P$ & & Odds ratio & $95 \% \mathrm{Cl}$ & $\mathrm{P}$ \\
\hline Age (per 10 years) & 0.000 & 2.760 & $2.037-3.738$ & 0.000 & 0.000 & 2.084 & $1.737-2.502$ & 0.000 & 0.000 & 3.478 & $2.648-4.570$ & 0.000 \\
\hline Sex (male) & 0.015 & & & & NS & & & & NS & & & \\
\hline Hypertension & 0.000 & 3.465 & $1.991-6.031$ & 0.000 & 0.000 & 1.611 & $1.120-2.315$ & 0.010 & 0.000 & 1.637 & $1.045-2.564$ & 0.031 \\
\hline Diabetes mellitus & 0.138 & & & & 0.035 & 1.914 & $1.092-3.355$ & 0.023 & NS & & & \\
\hline Hyperlipidemia & 0.171 & & & & 0.159 & & & & NS & & & \\
\hline Alcohol & 0.032 & 2.494 & $1.392-4.466$ & 0.002 & NS & & & & NS & & & \\
\hline Smoking & 0.018 & 2.302 & $1.161-4.565$ & 0.017 & 0.100 & & & & 0.121 & & & \\
\hline $\begin{array}{l}\text { Uric acid (per mg } \\
\text { per } 100 \mathrm{ml} \text { ) }\end{array}$ & 0.000 & & & & 0.150 & & & & 0.019 & 1.167 & $1.001-1.361$ & 0.048 \\
\hline
\end{tabular}


relative risk of stroke associated with smoking was 1.5 (95\% CI= 1.4-1.6). ${ }^{30}$ Subarachnoid hemorrhage was most clearly associated with smoking, and cerebral infarction was almost twice as likely in smokers compared with non-smokers. Although smoking may be more strongly related to atherogenic strokes rather than small vessel disease, ${ }^{31}$ the smoking-associated increased risk was found for lacunar infarction. ${ }^{32,33}$ Mannami et al. ${ }^{33}$ summarized several plausible mechanisms for smoking-related risk of stroke such as hypercoagulable states, reduced blood flow, reduced HDL cholesterol and direct injury to endothelial cells. The ARIC Study showed that current smoking and hypertension both almost doubled the odds of SBI (1.88 for current smoking, 2.00 for hypertension). ${ }^{15}$ In a high-risk Japanese community-dwelling population, smoking status and systolic blood pressure were independent determinant of the number of SBI. ${ }^{34}$ This study also found an independent association of smoking with SBI. Of note, the higher prevalence of SBI in men was partly due to the fact that smoking habit in women was extremely low in our population.

In conclusion, age, hypertension, alcohol and smoking were considered to be the risk factors for SBI in community-dwelling people. We showed that higher prevalence of alcohol habit and smoking in men than in women rather than biological effects of sex resulted in apparent male predominance in SBI in our population. Therefore, modification of the lifestyle risk factors would prevent SBI particularly in men and even in women with personal habits such as alcohol consumption and cigarette smoking.

\section{CONFLICT OF INTEREST}

The authors declare no conflict of interest.

\section{ACKNOWLEDGEMENTS}

We express special thanks to $\mathrm{T}$ Muto and $\mathrm{K}$ Yamamoto for their technical assistance with the laboratory examinations and the MRI scanning, K Fukuda for valuable advice on the relationship between alcohol and SBI and $\mathrm{N}$ Kawahara-Ideno for registration of participants.

1 Appelros P, Stegmayr B, Terént A. Sex differences in stroke epidemiology: a systematic review. Stroke 2009; 40: 1082-1090.

2 Sacco RL, Boden-Albala B, Gan R, Chen X, Kargman DE, Shea S, Paik MC, Hauser WA. Stroke incidence among white, black, and Hispanic residents of an urban community: the Northern Manhattan Stroke Study. Am J Epidemiol 1998; 147: 259-268.

3 Mendelsohn ME, Karas RH. The protective effects of estrogen on the cardiovascular system. N Engl J Med 1999; 340: 1801-1811.

4 Perls TT, Alpert L, Fretts RC. Middle-aged mothers live longer. Nature 1997; 389: 133.

5 Zhang X, Shu XO, Gao YT, Yang G, Li H, Zheng W. Pregnancy, childrearing, and risk of stroke in Chinese women. Stroke 2009; 40: 2680-2684.

6 Lisabeth LD, Beiser AS, Brown DL, Murabito JM, Kelly-Hayes M, Wolf PA. Age at natural menopause and risk of ischemic stroke: the Framingham Heart Study. Stroke 2009; 40: 1044-1049.

7 Prabhakaran S, Wright CB, Yoshita M, Delapaz R, Brown T, DeCarli C, Sacco RL. Prevalence and determinants of subclinical brain infarction: the Northern Manhattan Study. Neurology 2008; 70: 425-430.

8 Vermeer SE, Koudstaal PJ, Oudkerk M, Hofman A, Breteler MM. Prevalence and risk factors of silent brain infarcts in the population-based Rotterdam Scan Study. Stroke 2002; 33: 21-25.

9 Shinkawa A, Ueda K, Kiyohara Y, Kato I, Sueishi K, Tsuneyoshi M, Fujishima M. Silent cerebral infarction in a community-based autopsy series in Japan. The Hisayama Study. Stroke 1995; 26: 380-385.

10 Price TR, Manolio TA, Kronmal RA, Kittner SJ, Yue NC, Robbins J, Anton-Culver H, O'Leary $\mathrm{DH}$. Silent brain infarction on magnetic resonance imaging and neurological abnormalities in community-dwelling older adults. The Cardiovascular Health Study. CHS Collaborative Research Group. Stroke 1997; 28: 1158-1164.

11 Das RR, Seshadri S, Beiser AS, Kelly-Hayes M, Au R, Himali JJ, Kase CS, Benjamin EJ, Polak JF, O'Donnell CJ, Yoshita M, D'Agostino Sr RB, DeCarli C, Wolf PA. Prevalence and correlates of silent cerebral infarcts in the Framingham offspring study. Stroke 2008; 39: 2929-2935.

12 Yao H, Takashima Y, Mori T, Uchino A, Hashimoto M, Yuzuriha T, Miwa Y, Sasaguri T. Hypertension and white matter lesions are independently associated with apathetic behavior in healthy elderly subjects: the Sefuri brain MRI study. Hypertens Res 2009; 32: 586-590.

13 Fukuda K, Yuzuriha T, Kinukawa N, Murakawa R, Takashima Y, Uchino A, Ibayashi S, lida $M$, Yao $H$, Hirano $M$. Alcohol intake and quantitative MRI findings among community dwelling Japanese subjects. J Neurol Sci 2009; 278: 30-34.

14 Sasaki M, Hirai T, Taoka T, Higano S, Wakabayashi C, Matsusue E, Ida M. Discriminating between silent cerebral infarction and deep white matter hyperintensity using combinations of three types of magnetic resonance images: a multicenter observer performance study. Neuroradiology 2008; 50: 753-758.

15 Bokura H, Kobayashi S, Yamaguchi S. Distinguishing silent lacunar infarction from enlarged Virchow-Robin spaces: a magnetic resonance imaging and pathological study. J Neurol 1998; 245: 116-122.

16 Ohmine T, Miwa Y, Yao H, Yuzuriha T, Takashima Y, Uchino A, Takahashi-Yanaga F, Morimoto S, Maehara Y, Sasaguri T. Association between arterial stiffness and cerebral white matter lesions in community-dwelling elderly subjects. Hypertens Res 2008; $\mathbf{3 1}$ : 75-81.

17 Fazekas F, Kleinert R, Offenbacher H, Schmidt R, Kleinert G, Payer F, Radner H, Lechner H. Pathologic correlates of incidental MRI white matter signal hyperintensities. Neurology 1993; 43: 1683-1689.

18 Howard G, Wagenknecht LE, Cai J, Cooper L, Kraut MA, Toole JF. Cigarette smoking and other risk factors for silent cerebral infarction in the general population. Stroke 1998; 29: 913-917.

19 Kobayashi S, Okada K, Koide H, Bokura H, Yamaguchi S. Subcortical silent brain infarction as a risk factor for clinical stroke. Stroke 1997; 28: 1932-1939.

20 ESHRE Capri Workshop Group. Hormones and cardiovascular health in women. Hum Reprod Update 2006; 12: 483-497.

21 Humphries KH, Westendorp IC, Bots ML, Spinelli JJ, Carere RG, Hofman A, Witteman JC. Parity and carotid artery atherosclerosis in elderly women: the Rotterdam Study. Stroke 2001; 32: 2259-2264.

22 Skilton MR, Sérusclat A, Begg LM, Moulin P, Bonnet F. Parity and carotid atherosclerosis in men and women: insights into the roles of childbearing and child-rearing. Stroke 2009; 40: 1152-1157.

23 Vermeer SE, Den Heijer T, Koudstaal PJ, Oudkerk M, Hofman A, Breteler MM. Incidence and risk factors of silent brain infarcts in the population-based Rotterdam Scan Study. Stroke 2003; 34: 392-396.

24 Vermeer SE, Longstreth Jr WT, Koudstaal PJ. Silent brain infarcts: a systematic review. Lancet Neurol 2007: 6: 611-619.

25 Chen X, Wen W, Anstey KJ, Sachdev PS. Prevalence, incidence, and risk factors of lacunar infarcts in a community sample. Neurology 2009; 73: 266-272.

26 Mukamal KJ, Longstreth Jr WT, Mittleman MA, Crum RM, Siscovick DS. Alcohol consumption and subclinical findings on magnetic resonance imaging of the brain in older adults: the cardiovascular health study. Stroke 2001; 32: 1939-1946.

27 Ding J, Eigenbrodt ML, Mosley Jr TH, Hutchinson RG, Folsom AR, Harris TB, Nieto FJ. Alcohol intake and cerebral abnormalities on magnetic resonance imaging in a community-based population of middle-aged adults: the Atherosclerosis Risk in Communities (ARIC) study. Stroke 2004; 35: 16-21.

28 Shibuya A, Yoshida A. Frequency of the atypical aldehyde dehydrogenase-2 gene (ALDH2(2)) in Japanese and Caucasians. Am J Hum Genet 1988; 43: 741-743.

29 Tanizaki Y, Kiyohara Y, Kato I, Iwamoto H, Nakayama K, Shinohara N, Arima H, Tanaka K, Ibayashi S, Fujishima M. Incidence and risk factors for subtypes of cerebral infarction in a general population: the Hisayama study. Stroke 2000; 31: 2616-2622.

30 Shinton R, Beevers G. Meta-analysis of relation between cigarette smoking and stroke. BMJ 1989; 298: 789-794.

31 Mast H, Thompson JL, Lin IF, Hofmeister C, Hartmann A, Marx P, Mohr JP, Sacco RL. Cigarette smoking as a determinant of high-grade carotid artery stenosis in Hispanic, black, and white patients with stroke or transient ischemic attack. Stroke 1998; 29: 908-912.

32 You R, McNeil JJ, O'Malley HM, Davis SM, Donnan GA. Risk factors for lacunar infarction syndromes. Neurology 1995; 45: 1483-1487.

33 Mannami T, Iso H, Baba S, Sasaki S, Okada K, Konishi M, Tsugane S, Japan Public Health Center-Based Prospective Study on Cancer and Cardiovascular Disease Group. Cigarette smoking and risk of stroke and its subtypes among middle-aged Japanese men and women: the JPHC Study Cohort I. Stroke 2004; 35: 1248-1253.

34 Eguchi K, Kario K, Hoshide S, Hoshide Y, Ishikawa J, Morinari M, Hashimoto T, Shimada K. Smoking is associated with silent cerebrovascular disease in a high-risk Japanese community-dwelling population. Hypertens Res 2004; 27: 747-754. 